Research Article

\title{
Evaluation of Retinal Structure and Optic Nerve Function Changes in Multiple Sclerosis: Longitudinal Study with 1-Year Follow-Up
}

\author{
Riwanti Estiasari $\left(\mathbb{D},{ }^{1}\right.$ Adisresti Diwyacitta, ${ }^{1}$ Muhammad Sidik, ${ }^{2}$ Ni Nengah Rida Ariarini, ${ }^{1}$ \\ Freddy Sitorus, ${ }^{1}$ Saraf Shafa Marwadhani, ${ }^{1}$ Kartika Maharani, ${ }^{1}$ Darma Imran, ${ }^{1}$ \\ Reza Aditya Arpandy, ${ }^{1}$ David Pangeran, ${ }^{1}$ and Manfaluthy Hakim ${ }^{1}$ \\ ${ }^{1}$ Department of Neurology, Cipto Mangunkusumo General Hospital, Universitas Indonesia, Jakarta, Indonesia \\ ${ }^{2}$ Department of Ophtalmology, Cipto Mangunkusumo General Hospital, Universitas Indonesia, Jakarta, Indonesia \\ Correspondence should be addressed to Riwanti Estiasari; riwanti.estiasari04@ui.ac.id
}

Received 20 February 2021; Revised 23 May 2021; Accepted 31 May 2021; Published 17 June 2021

Academic Editor: Carol Milligan

Copyright (c) 2021 Riwanti Estiasari et al. This is an open access article distributed under the Creative Commons Attribution License, which permits unrestricted use, distribution, and reproduction in any medium, provided the original work is properly cited.

\begin{abstract}
Background. Multiple sclerosis (MS) is an autoimmune disease characterized by inflammation and demyelination of the central nervous system which often involves the optic nerve even though only $20 \%$ of the patients experience optic neuritis (ON). Objective. This study aims to compare the retinal structure and optic nerve function between patients with MS and healthy controls (HCs), evaluate optic nerve alterations in MS over 1-year follow-up, and analyze its correlations with disease duration, number of relapses, degree of disability, and different subtypes. Methods. This is a prospective cohort study involving 58 eyes of MS patients. Optic nerve function was evaluated with best-corrected visual acuity (BCVA), contrast sensitivity, and P100 latency, while the retinal structure was evaluated from the GCIPL and RNFL thickness measured with optical coherence tomography (OCT) and fundus photography. Results. The MS group had lower BCVA $(p=0.001)$, contrast sensitivity $(p<0.001)$, mean GCIPL thickness $(p<0.001)$, and mean RNFL thickness $(p<0.001)$ than HC. At 6 and 12 months of observations, GCIPL and RNFL (nasal quadrant) of MS patients decreased significantly ( $p=0.007$ and $p=0.004$, respectively). Disease duration and the number of relapses correlated with delayed P100 latency $(r=-0.61, p<0.001$ and $r=-0.46, p=0.02)$. GCIPL and RNFL in the SPMS subtype were thinner than in RRMS. Conclusions. The retinal structure and optic nerve function of MS patients are worse than those of normal individuals. GCIPL and RNFL thinning occurs at 6 and 12 months but do not correlate with disease duration, the number of relapses, and degree of disability.
\end{abstract}

\section{Introduction}

Multiple Sclerosis (MS) is a chronic immune-mediated disease of the central nervous system affecting 2.5 million people globally [1]. The prevalence of MS in Indonesia is $0-5$ per 100.000 individuals with the highest incidence in women aged 20 to 40 [2-4]. Although considered a rare disease, MS is the leading cause of nontraumatic neurological disability in the young population [1]. MS data in Indonesia are rarely reported. A previous study in Indonesia demonstrated that the clinical characteristics of
MS which were generally similar to those of MS patients in Western countries. The most subtypes found in Indonesia are also RRMS [2].

Inflammatory and demyelinating pathology in MS frequently involves the visual pathway [5]. Despite only $20 \%$ of MS patients showing optic neuritis (ON), an autopsy study revealed that $94-99 \%$ of MS patients have detectable optic nerve lesions even in the absence of any visual symptoms $[6,7]$. Given its susceptibility to damage in the early stage of the disease, visual measures have been a focus of recent studies to evaluate the progression of MS [8-10]. 
Numerous tools and techniques have been developed to portray the structure and function of the optic nerve that can provide clinically meaningful information of MS [10-12]. The structural composition of the retina can be observed using Optical Coherence Tomography (OCT), while the function may be evaluated through the Visual Evoked Potential (VEP) test $[8,11]$. According to The 25-Item National Eye Institute Visual Functioning Questionnaire (NEI-VFQ25) and 10-Item Neuro-Ophthalmic Supplement, the most common visual symptoms in MS include a decrease in visual acuity and contrast sensitivity [12]. The latter can be observed using the Pelli-Robson charts.

The pathogenesis of optic neuritis is not well understood. A recent study suggests that axonal damage, not related to demyelination, is the major contributor to permanent visual loss [13]. It is reflected in the thickness of the retinal nerve fiber layer (RNFL), the innermost retinal layer which comprises nonmyelinated axons of the retinal ganglion cells (RGCs) [13]. OCT offers $75 \%$ sensitivity and $81 \%$ specificity to monitor axonal and neuronal loss in MS in terms of RNFL thickness [14]. Studies showed that RNFL thickness is lower in MS patients than that in normal individuals and would decrease by $10-40 \mathrm{~mm}$ within 3-6 months after an acute $\mathrm{ON}$ episode $[11,15,16]$. Recent findings suggest that the ganglion cell inner plexiform layer (GCIPL) is more accurate in depicting the progression of MS. It also demonstrates a better correlation with visual dysfunction as it is less sensitive to neuroinflammation $[17,18]$.

VEP serves as an approach to assess the function of the optic nerve by recording electrical changes following stimulation [11]. The sensitivity and specificity of VEP in MS are $60.7 \%$ and $80.5 \%$, respectively. Another study reported higher VEP sensitivity of $90 \%$ [19]. A typical finding of MS during VEP measurement is prolonged P100 latency regardless of any prior episode of ON [11].

Previous studies have investigated the association between the retinal structure and optic nerve function with disease duration, number of relapses, subtype, and degree of disability in MS. As discussed by Balk et al., the thinning of RNFL and GCIPL is influenced by disease duration and occurs predominantly in the early stage of the disease [20]. In another study, a significant association was only found between the temporal RNFL and disease duration [21]. The progression of MS seems to follow relapse, but no evidence proves that relapse causes progression [22]. A multicenter study showed that RNFL thickness is lower in secondary progressive type (SPMS) than relapsing-remitting type (RRMS) [23]. RNFL, GCIPL, and contrast sensitivity are also known to affect the Expanded Disability Status Scale (EDSS) score with GCIPL having the strongest association with EDSS [24-26]. RNFL thickness lower than $88 \mu \mathrm{m}$ is associated with a threefold increased risk of EDSS progression in 3 years [27].

In our previous study, we found that GCIPL, RNFL, and visual acuity of patients with Neuromyelitis Optica Spectrum Disorders (NMOSD) are lower than those of patients with MS [28]. The three parameters were also inversely correlated with disease duration, while the number of relapses was the only parameter that showed no association with other variables. The limitation of this study is the lack of healthy controls.

Given the information, the aims of this study are (1) to compare the retinal structure and optic nerve function between multiple sclerosis patients and healthy individuals, (2) to evaluate the alteration of retinal structure and optic nerve function of MS patients in the 1-year follow-up, and (3) to explore the association between visual parameters with disease duration, the number of relapses, and degree of disability in MS.

\section{Materials and Methods}

2.1. Design and Participants. This was a prospective cohort study with a duration of 1 year, starting from September 2018 to April 2019. Participants were recruited from the Neurology clinic at Dr. Cipto Mangunkusumo General Hospital using a consecutive nonrandom sampling method. Eligible patients must sign a written consent before participating in the study. This research was approved by the Ethics Committee of the Faculty of Medicine Universitas Indonesia and was funded by Hibah PITTA 2018 DRPM Universitas Indonesia (PITTA/214/FK/2018).

A total of 102 eyes from 32 patients with MS and 22 normal individuals were evaluated at baseline, 6 months, and 12 months. We used our previous research data of 110 normal individuals (220 eyes) for VEP controls [28]. All eyes were classified into three groups: multiple sclerosis (58 eyes), healthy control for OCT (44 eyes), and healthy controls for VEP (110 eyes).

2.2. Inclusion and Exclusion Criteria. Inclusion criteria were (1) age 18-60 years old, (2) providing a written consent containing all tests included in the study, and (3) meeting the diagnostic criteria for MS (exposed group) or having bestcorrected visual acuity (BCVA) of 20/20 (healthy control).

Exclusion criteria were (1) experiencing ON within 6 months before enrollment, (2) having other types of optic neuropathy (ischemic, infection, hereditary, compression/ infiltration, toxic, and trauma), (3) having other ophthalmological conditions (refractive errors $>6$ diopters (D), glaucoma), (4) having diabetes mellitus, grade II hypertension, or obesity (BMI $\geq 40 \mathrm{~kg} / \mathrm{m}^{2}$ ), and (5) having a history of taking drugs with ocular side effects, such as hydroxychloroquine and ethambutol.

2.3. Data Collection. Anamnesis, physical examination, and neurological examination were performed on all participants. Confounding illnesses and conditions were excluded during history taking and physical examination. Intraocular pressure (IOP) was measured on both eyes with a Schiotz tonometer, using pantocaine $0.5 \%$ as an anesthetic. Demographic data (sex and age), disability status (EDSS), and the number of relapses were recorded. We analyze the optic nerve structure using OCT and retinal fundus image, and for function, we used the VEP (P-100 latency).

Best-corrected visual acuity (BCVA) was assessed using the Snellen chart, and contrast sensitivity was measured 
monocularly using the Pelli-Robson chart. All measurements were repeated at 6 and 12 months. Some of the data were obtained from the patients' medical records since BCVA, contrast sensitivity, OCT, VEP, and fundus photography were part of our routine clinical evaluations at the clinic. For the VEP measurement of healthy control, we used secondary data from our previous research by Wijaya et al. [29].

\subsubsection{Optical Coherence Tomography and Retinal Fundus} Images. We used Cirrus HD OCT 5000 with optic disc cube $200 \times 200$ protocol and macular cube $512 \times 128$ protocol. RNFL thickness measurement was done by a certified optician. Participants were scanned in pupillary dilation which was obtained with tropicamide $1 \%$. The fundus photograph was taken for each eye using the Kowa VX-10 fundus camera. The resulting images were masked and reviewed by 2 experts based on the agreed operational definition. If any discrepancy happened between the 2 reviewers, a third person would make the decision.

2.3.2. Visual Evoked Potential. VEP was measured monocularly on both eyes with Caldwell Sierra Summit and 24 inch LCD ViewSonic VT2216-L monitor which had been calibrated digitally. The VEP was interpreted by a neurophysiologist.

2.4. Statistical Analysis. In the descriptive analysis, quantitative variables are presented in mean and standard deviation for normally distributed data or median and range for nonnormally distributed data. Statistical analysis was carried out using SPSS version 20.0. Numerical variables are tested for normality before proceeding with further analysis. In variables of which the participants were measured multiple times, data were analyzed using repeated ANOVA if distributed normally. Otherwise, the Friedman test was used. We used the T-independent test to compare mean scores of the same variable from two different groups for normally distributed data or the Mann-Whitney for nonnormally distributed data. To test the correlation, we used Pearson's correlation coefficient for normally distributed data, otherwise Spearman's correlation formula. To assess qualitative variables and determine the agreement between two reviewers, we used the kappa coefficient. Kappa coefficient is acceptable if the value is $>0.8$ [30]. Statistical significance was established at $p<0.05$.

\section{Results}

3.1. Baseline Characteristics. Initially, 32 MS patients were recruited in the study. Four eyes were excluded due to severe nystagmus and the other four had a high refractive error of $\geq 6$ diopters, yielding a total of 58 eyes. Besides, there is one missing data on GCIPL in healthy controls for OCT (HC OCT) due to recording error. For VEP healthy control (HC VEP), we used data from our previous research by Wijaya which included 110 normal participants (220 eyes) [29]. The baseline characteristics of both MS and healthy controls are depicted in Table 1. In general, all groups are homogeneous, except for the sex ratio between the MS group and HC VEP $(p<0.001)$. The ratio of male: female is $1: 5$ with ethnic distribution, Javanese (26.7\%), Chinese descent (23.3\%), Sundanese 10\%, Manado, Minang, Betawi 6.7\%, and Batak $3 \%$. There was no difference in the percentage of optic neuritis occurrences between these ethnic groups.

We identified 58 eyes at the entry visit (baseline), but only 34 eyes could be evaluated at 6 months. One participant was excluded for being 60 years by the time of the second measurement, and the rest were lost to follow-up. At 12 months, only 10 patients (20 eyes) returned for the last measurement. One patient ( 2 eyes) died, and 6 patients (12 eyes) could not make it to the hospital.

\subsection{Comparison of the Retinal Structure and Optic Nerve} Function between MS Patients and Healthy Controls. We found that the baseline retinal structure and optic nerve function in MS are lower than those in healthy controls, marked by significantly thinner GCIPL and RNFL (in all sectors except the nasal quadrant) and longer P100 latency. Optic atrophy was only seen in the MS group with a percentage of $42.6 \%$. See Table 2.

\subsection{Alteration of Retinal Structure and Optic Nerve Function} in MS Patients at 6 and 12 Months. Table 3 summarizes the functional evaluations obtained at baseline, 6 months, and 12 months. No significant changes were observed in BCVA and contrast sensitivity from all time. Meanwhile, P100 latency was significantly longer at 12 months compared to 6 months $(124.43 \pm 19.13$ vs $110.07 \pm 19.97, p<0.001)$ and baseline $(124.43 \pm 19.13$ vs $116.63 \pm 19.19, p=0.002)$.

At baseline, 23 out of 58 eyes presented with optic atrophy on their fundus photographs. Six months later, among 34 eyes being scanned, six previously normal eyes worsened into optic atrophy, two of which had a history of ON, and another two never had ON nor relapses. These findings were captured from four female patients aged 27-47 years who had been diagnosed with RRMS for 6 months to 6 years. Their EDSS score ranged from 1.0 to 6.0. On the other side, one eye exhibited recovery from optic atrophy which was obtained from a 22 -year-old female in the absence of prior ON. She had been diagnosed with RRMS for 7.5 years and experienced 3 relapses. Her EDSS score was 2.5. At 12 months, one previously normal eye developed optic atrophy which was observed from a 29 -year-old female with RRMS and no history of ON. She had been diagnosed with MS for 6 years with 6 relapses and an EDSS score of 1.5.

As seen in Table 4, the present study shows a decrease in GCIPL thickness in MS patients over 1 year with the most prominent change observed between the baseline and the 12 -month evaluation $(70.89 \pm 10.60$ vs $67.95 \pm 11.12$, $p=0.01)$. Statistically significant thinning was found in all quadrants, except the superior and superotemporal quadrant (Figure 1). In the superonasal quadrant, a significant difference was found between the 6- and 12-month 
TABLE 1: Demographic and clinical data of multiple sclerosis (MS), healthy controls for OCT (HC OCT), and healthy controls for VEP (HC VEP).

\begin{tabular}{lcccc}
\hline Variable & MS $(N=32)$ & HC OCT $(N=22)$ & $p$ & HC VEP $(N=110)$ \\
\hline$N(\%)$ & & & & \\
Sex; $N(\%)$ & $5(15.6 \%)$ & $5(22.7 \%)$ & $0.72^{\mathrm{a}}$ & $55(50 \%)$ \\
(i) Male & $27(84.4 \%)$ & $17(77.3 \%)$ & & $55(50 \%)$ \\
(ii) Female & & & $0.42^{\mathrm{b}}$ & $29.0(19.0-55.0)$ \\
Median (range) & $29.5(19.0-59.0)$ & $28.5(20.0-57.0)$ & - & - \\
Age (years) & $3.72(0.50-15.94)$ & - & - & - \\
Disease duration (years) & $4.0(0.0-13.0)$ & - & - & $0.24^{\mathrm{b}}$ \\
Relapse $(n)$ & $3.0(0.0-8.0)$ & - & & - \\
EDSS & & - & & \\
\hline
\end{tabular}

EDSS: Expanded Disability Status Scale. ${ }^{a}$ Fisher's test. ${ }^{b}$ Mann-Whitney test. ${ }^{c}$ Chi-square test.

TABle 2: Comparison of BCVA, contrast sensitivity, GCIPL, RNFL, P100 latency, and fundus image between MS and healthy controls (HCs).

\begin{tabular}{|c|c|c|c|c|c|}
\hline Variable & $n$ & MS & $n$ & $\mathrm{HC}$ & $p$ value \\
\hline \multicolumn{6}{|l|}{ Median (range) } \\
\hline BCVA (logmar) & 58 & $0.00(0.00-1.77)$ & 44 & $0.00(0.00-0.10)$ & \multirow{2}{*}{$\begin{array}{c}0.001^{b} \\
0.00000000028^{b}\end{array}$} \\
\hline Contrast sensitivity & 53 & $1.65(0.90-1.95)$ & 44 & $1.95(1.65-1.95)$ & \\
\hline \multicolumn{6}{|c|}{ Mean \pm standard deviation } \\
\hline \multicolumn{6}{|c|}{ GCIPL } \\
\hline Mean & 54 & $70.89 \pm 10.60$ & 43 & $84.72 \pm 2.99$ & \multirow{7}{*}{$\begin{array}{c}0.00000000000036^{\mathrm{a}} \\
0.000000000083^{\mathrm{b}} \\
0.000000000033^{\mathrm{b}} \\
0.0000000027^{\mathrm{b}} \\
0.000000000022^{\mathrm{a}} \\
0.0000000021^{\mathrm{b}} \\
0.00000000006^{\mathrm{b}}\end{array}$} \\
\hline Superior & 54 & $71.11 \pm 11.44$ & 43 & $86.05 \pm 2.96$ & \\
\hline Superonasal & 54 & $71.00 \pm 12.51$ & 43 & $87.58 \pm 3.56$ & \\
\hline Inferonasal & 54 & $70.28 \pm 12.74$ & 43 & $85.53 \pm 3.99$ & \\
\hline Inferior & 54 & $69.35 \pm 11.06$ & 43 & $82.30 \pm 4.07$ & \\
\hline Inferotemporal & 54 & $71.83 \pm 10.12$ & 43 & $83.72 \pm 3.74$ & \\
\hline Superotemporal & 54 & $71.15 \pm 9.82$ & 43 & $83.53 \pm 4.00$ & \\
\hline \multicolumn{6}{|l|}{$R N F L$} \\
\hline Mean & 54 & $88.48 \pm 14.08$ & 44 & $102.50 \pm 7.76$ & \multirow{6}{*}{$\begin{array}{c}0.000000016^{\mathrm{a}} \\
0.000008^{\mathrm{a}} \\
0.12^{\mathrm{a}} \\
0.00000000099^{\mathrm{a}} \\
0.00003^{\mathrm{a}} \\
0.0000000098^{\mathrm{b}}\end{array}$} \\
\hline Superior & 54 & $115.69 \pm 21.95$ & 44 & $132.36 \pm 12.34$ & \\
\hline Nasal & 54 & $68.17 \pm 10.60$ & 44 & $71.73 \pm 11.58$ & \\
\hline Inferior & 54 & $110.28 \pm 20.78$ & 44 & $134.93 \pm 15.14$ & \\
\hline Temporal & 54 & $59.37 \pm 16.82$ & 44 & $70.80 \pm 7.90$ & \\
\hline P100 latency & 54 & $116.63 \pm 19.19$ & 220 & $100.62 \pm 5.73$ & \\
\hline \multicolumn{6}{|l|}{ Fundus image; $N(\%)$} \\
\hline Normal & & $31(57.4)$ & & $44(100)$ & \multirow{2}{*}{$0.00000075^{c}$} \\
\hline Optic atrophy & & $23(42.6)$ & & $0(0,0)$ & \\
\hline
\end{tabular}

BCVA: best-corrected visual acuity; GCIPL: ganglion cell inner plexiform layer; RNFL: retinal nerve fiber layer. ${ }^{a}$ T-independent test, ${ }^{\mathrm{b}} \mathrm{Mann}-\mathrm{Whitney}$ test, ${ }^{\mathrm{c}} \mathrm{Chi}$-square test.

TABle 3: BCVA, contrast sensitivity, and P100 latency value of MS eyes at baseline, 6 months, and 12 months.

\begin{tabular}{|c|c|c|c|c|c|c|c|}
\hline \multirow{2}{*}{ Visual parameter } & \multicolumn{6}{|c|}{ MS } & \multirow{2}{*}{$p$} \\
\hline & $n$ & Baseline & $n$ & 6 months & $n$ & 12 months & \\
\hline BCVA (logmar) & 58 & $0.0(0.00-1.77)$ & 34 & $0.00(0.00-1.85)$ & 20 & $0.00(0.00-1.78)$ & $\begin{array}{l}0.50^{\mathrm{a}} \\
0.61^{\mathrm{b}} \\
0.23^{\mathrm{c}}\end{array}$ \\
\hline Contrast sensitivity & 53 & $1.65(0.90-1.95)$ & 33 & $1.65(1.05-1.95)$ & 19 & $1.65(1.20-1.95)$ & $\begin{array}{l}0.51^{\mathrm{a}} \\
0.59^{\mathrm{b}} \\
0.16^{\mathrm{c}}\end{array}$ \\
\hline P100 latency & 54 & $116.63 \pm 19.19$ & 33 & $110.07 \pm 19.97$ & 19 & $124.43 \pm 19.13$ & $\begin{array}{c}0.14^{\mathrm{a}} \\
\mathbf{0 . 0 0 0 2 3 ^ { \mathrm { b } }} \\
\mathbf{0 . 0 0 2}\end{array}$ \\
\hline
\end{tabular}

BCVA= best-corrected visual acuity. ${ }^{\mathrm{a}} 0$ vs 6 months, ${ }^{\mathrm{b}} 6$ vs 12 months, ${ }^{\mathrm{c}} 0$ vs 12 months. The Friedman test was followed by the Wilcoxon test.

evaluations $(69.88 \pm 12.65$ vs $68.05 \pm 12.90, p=0.04)$. In the inferonasal quadrant, the differences were noted between the 0 - and 6-month evaluations (70.28 \pm 12.74 vs $68.65 \pm 12.65$, $p=0.04)$ and 0 - and 12-month evaluation (70.28 \pm 12.74 vs $67.41 \pm 13.75, p=0.01)$. In the inferior and inferotemporal quadrant, a significant difference was observed between the 
TABLE 4: Comparison of baseline, 6 months, and 12 months of evaluation on GCIPL and RNFL thickness of MS eyes.

\begin{tabular}{|c|c|c|c|c|c|c|c|c|c|}
\hline \multirow{2}{*}{ Variable } & \multicolumn{6}{|c|}{ MS } & \multicolumn{3}{|c|}{$p$} \\
\hline & $n$ & Baseline & $n$ & 6 months & $n$ & 12 months & 0 vs 6 & 6 vs 12 & 0 vs 12 \\
\hline \multicolumn{10}{|c|}{ Mean \pm standard deviation } \\
\hline \multicolumn{10}{|c|}{ GCIPL } \\
\hline Mean & 54 & $70.89 \pm 10.60$ & 34 & $69.38 \pm 10.87$ & 20 & $67.95 \pm 11.12$ & $0.105^{\mathrm{a}}$ & $0.150^{\mathrm{a}}$ & $0.007^{\mathrm{a}}$ \\
\hline Superior & 54 & $71.11 \pm 11.44$ & 34 & $70.32 \pm 11.07$ & 20 & $68.82 \pm 11.59$ & $1.000^{\mathrm{b}}$ & $0.579^{\mathrm{b}}$ & $0.619^{\mathrm{b}}$ \\
\hline Superonasal & 54 & $71.00 \pm 12.51$ & 34 & $69.88 \pm 12.65$ & 20 & $68.05 \pm 12.90$ & $0.294^{\mathrm{a}}$ & $0.038^{\mathrm{a}}$ & $0.053^{\mathrm{a}}$ \\
\hline Inferonasal & 54 & $70.28 \pm 12.74$ & 34 & $68.65 \pm 12.65$ & 20 & $67.41 \pm 13.75$ & $0.039^{\mathrm{a}}$ & $0.495^{\mathrm{a}}$ & $0.012^{\mathrm{a}}$ \\
\hline Inferior & 54 & $69.35 \pm 11.06$ & 34 & $68.18 \pm 11.02$ & 20 & $66.45 \pm 11.68$ & $0.394^{\mathrm{a}}$ & $0.208^{\mathrm{a}}$ & $0.046^{\mathrm{a}}$ \\
\hline Inferotemporal & 54 & $71.83 \pm 10.12$ & 34 & $70.06 \pm 10.46$ & 20 & $68.36 \pm 10.49$ & $0.108^{\mathrm{a}}$ & $0.284^{\mathrm{a}}$ & $0.012^{\mathrm{a}}$ \\
\hline Superotemporal & 54 & $71.15 \pm 9.82$ & 34 & $69.15 \pm 10.25$ & 20 & $69.00 \pm 10.37$ & $0.080^{\mathrm{b}}$ & $1.000^{\mathrm{b}}$ & $0.389^{\mathrm{b}}$ \\
\hline \multicolumn{10}{|l|}{ RNFL } \\
\hline Mean & 54 & $88.48 \pm 14.08$ & 34 & $87.62 \pm 14.12$ & 20 & $86.50 \pm 14.22$ & $1.000^{\mathrm{b}}$ & $1.000^{\mathrm{b}}$ & $0.836^{\mathrm{b}}$ \\
\hline Superior & 54 & $115.69 \pm 21.95$ & 34 & $115.91 \pm 25.69$ & 20 & $114.50 \pm 22.71$ & $1.000^{\mathrm{b}}$ & $1.000^{\mathrm{b}}$ & $1.000^{\mathrm{b}}$ \\
\hline Nasal & 54 & $68.17 \pm 10.60$ & 34 & $67.79 \pm 9.66$ & 20 & $68.50 \pm 8.77$ & $0.933^{\mathrm{a}}$ & $0.093^{\mathrm{a}}$ & $0.004^{\mathrm{a}}$ \\
\hline Inferior & 54 & $110.28 \pm 20.78$ & 34 & $107.62 \pm 22.09$ & 20 & $105.45 \pm 22.25$ & $1.000^{\mathrm{a}}$ & $0.368^{\mathrm{a}}$ & $0.727^{\mathrm{a}}$ \\
\hline Temporal & 54 & $59.37 \pm 16.82$ & 34 & $59.32 \pm 15.61$ & 20 & $57.85 \pm 17.41$ & $0.376^{\mathrm{b}}$ & $0.537^{\mathrm{b}}$ & $1.000^{\mathrm{b}}$ \\
\hline \multicolumn{10}{|c|}{ Gambaran fundus; $N$ (\%) } \\
\hline Normal & $31(57.4)$ & - & $14(41.2)$ & - & $8(36.4)$ & - & & & \\
\hline Atrofi papil & $23(42.6)$ & - & $20(58.8)$ & - & $14(63.6)$ & - & & $0.719^{\circ}$ & $0.096^{c}$ \\
\hline
\end{tabular}

GCIPL: ganglion cell inner plexiform layer; RNFL: retinal nerve fiber layer, ${ }^{\mathrm{a}}$ Friedman test followed by Wilcoxon test, ${ }^{\mathrm{b}}$ Repeated ANOVA followed by Bonferonni test, ${ }^{\mathrm{C}} \mathrm{Chi}$-square test.

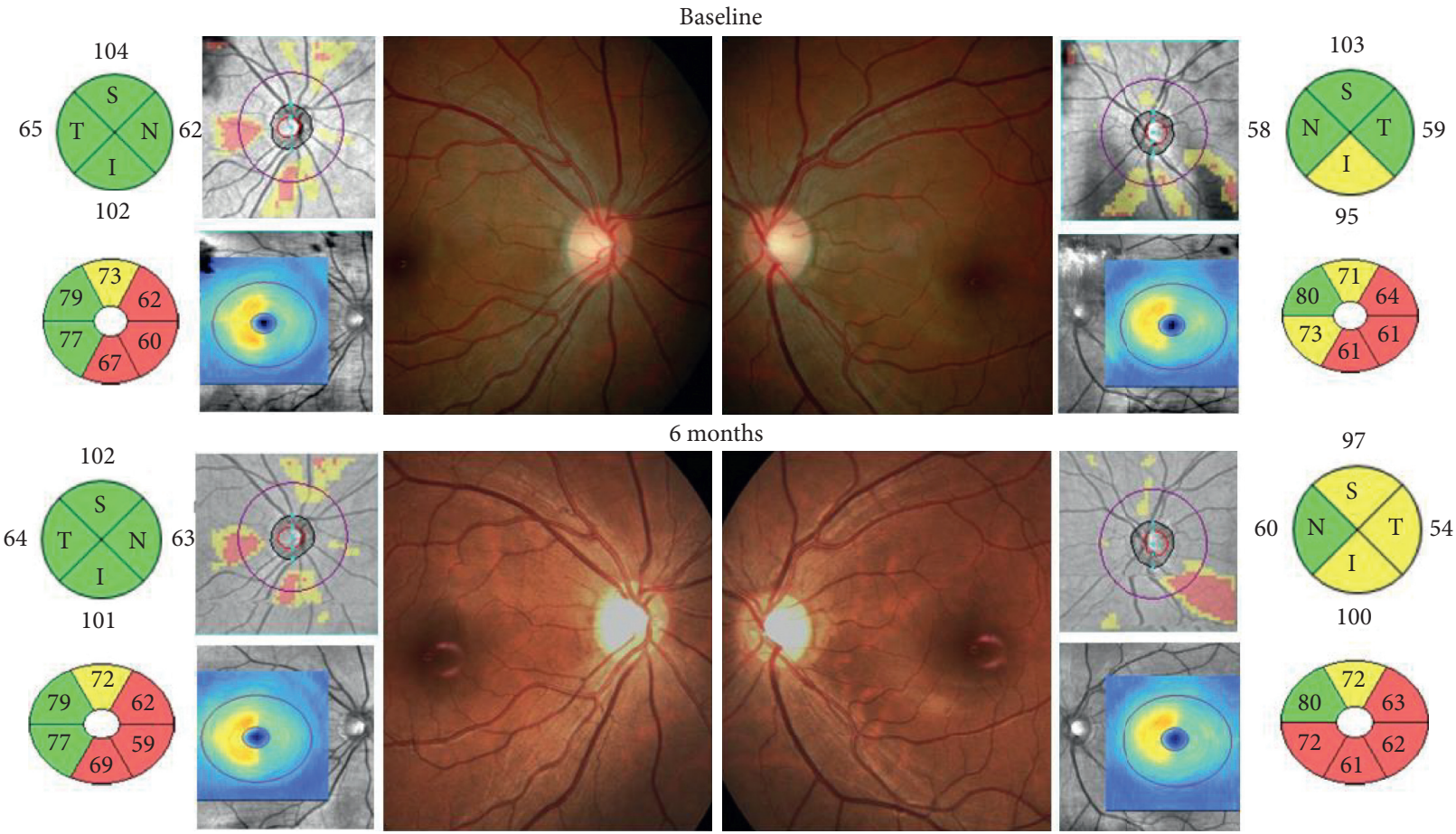

FIGURE 1: 28-year-old female with SPMS. The RNFL and GCIPL become thinner, optic nerve head appeared paler, but contrast sensitivity was stable during the 6-month observation.

0 - and 12-month evaluations $(69.35 \pm 11.06$ vs $66.45 \pm 11.68$, $p=0.046 ; 71.83 \pm 10.12$ vs $68.36 \pm 10.49, p=0.01)$.

In contrast to GCIPL, a significant difference in RNFL thickness was only seen in the nasal quadrant between the 0 and 12-month evaluations, where the thickness increased at 12 months after a slight decrease at 6 months.
3.4. Correlation Analysis of Visual Parameters, Disease Duration, and Number of Relapses. The number of relapses showed a negative correlation with GCIPL thickness of the superonasal quadrant $(p=0.03, r=-0.29)$, inferonasal $(p=0.03, r=-0.30)$, and mean RNFL thickness $(p=0.04$, $r=-0.28)$. EDSS score showed a negative correlation with 
mean GCIPL, the superonasal, inferonasal, and inferior quadrant thickness. In addition, EDSS was inversely correlated with the RNFL thickness of the inferior quadrant $(p=0.01, r=-0.34)$.

We found that the rates of change on P100 latency, RNFL thickness, and GCIPL thickness throughout the study were relatively stable. In correlation analysis, the rate of change on P100 latency was shown to have a negative correlation with disease duration $(r=-0.61, p<0.001)$ and the number of relapses $(r=-0.46, p=0.02)$.

\subsection{Comparison of the Retinal Structure and Optic Nerve} Function in MS Subtypes. Of all $32 \mathrm{MS}$ participants included in this study, 25 patients (78\%) belonged to the relapsingremitting subtype (RRMS) and 7 patients (22\%) to the secondary progressive subtype (SPMS). No significant difference was observed between RRMS and SPMS in terms of visual acuity, contrast sensitivity, P100 latency, and fundus image. GCIPL thickness on SPMS eyes was significantly thinner compared to RRMS eyes on average and in distinctive quadrants (see Table 5). Likewise, RNFL thickness was significantly lower in SPMS than RRMS, except for the nasal quadrant.

3.6. Comparison of Visual Parameters Based on the History of Optic Neuritis. In the present study, ON was reported in 17 eyes (MS-NO), while the other 41 eyes had no history of ON (MS-NNO). In MS-NO, no significant alteration was detected in almost all visual parameters over 1-year follow-up (visual acuity, contrast sensitivity, RNFL, P100 latency, and fundus image). The only meaningful change was observed in the inferonasal GCIPL thickness between 0 and 6 months, where it increased significantly $(67.62 \pm 16.99$ vs $70.33 \pm 17.23, p=0.02$ ). In terms of fundus photographs, the results of the MS-NO group were relatively stable throughout the study. There were initially 8 eyes with normal fundus images and 7 eyes with optic atrophy at baseline. At 6 months, 2 previously normal eyes developed optic atrophy.

In contrast to MS-NO, we observed significant differences of GCIPL as well as of P100 latency in MS-NNO throughout 1 year. P100 latency lengthened significantly at 12 months compared to baseline (128.20 \pm 18.96 vs $113.13 \pm 20.25, p=0.001)$ and 6 months $(128.20 \pm 18.96$ vs $114.70 \pm 17.44, p=0.02)$. With respect to GCIPL, significant changes were observed (1) between 0 and 6 months in the inferotemporal quadrant $(73.41 \pm 8.67$ vs $70.52 \pm 8.97$, $p=0.04)$ and superotemporal quadrant $(72.17 \pm 8.36$ vs $69.56 \pm 8.72, p=0.04$ ) and (2) between 0 and 12 months in the inferonasal quadrant $(71.12 \pm 11.20$ vs $65.19 \pm 10.65$, $p=0.024)$, inferior quadrant $(70.80 \pm 9.70$ vs $65.31 \pm 9,10$, $p=0.041)$, and mean GCIPL $(72.22 \pm 9.26$ vs $66.81 \pm 8.9$, $p=0.011)$.

In terms of fundus photographs, the ratio of normal and optic atrophy in the MS-NNO tended to decrease over time. At the start, 23 eyes had a normal fundus appearance while 16 eyes had optic atrophy. At 6 months, 2 patients (4 eyes) developed optic atrophy (see Table 6). They were 47- and 27year-old patients with RRMS. Their disease duration, number of relapses, and EDSS score were as follows: 6 months, 3 years; no relapse, 6 relapses; 1.0, 6.0. In contrast, there was 1 eye that recovered from optic atrophy to normal fundus. This eye was scanned from a 22-year-old female RRMS patient with 7.5 years of disease duration and 2.5 of EDSS score. At 12 months of evaluation, 1 eye developed optic atrophy which was evaluated from a 29 -year-old female with RRMS with 6 years of disease duration, 6 previous relapses, and 1.5 of EDSS score.

\section{Discussion}

4.1. Visual Parameters in MS and Healthy Controls. Our study shows that MS patients have significantly lower BCVA, contrast sensitivity, GCIPL, RNFL, and longer P100 latency than healthy controls which are consistent with previous studies [12, 24, 31]. GCIPL thinning among MS patients may be caused by a microscopic optic nerve inflammatory process, where the retinal ganglion cells (RGCs) are at greater risk for neurodegeneration. Thus, rates of GCIPL thinning are faster in patients exhibiting evidence of disease activity [32].

In our study, RNFL thinning was apparent in every sector except the nasal quadrant, corroborating a study by Pillay et al. [31] which suggests that the nasal quadrant is most resistant to RNFL loss. On the other hand, the temporal quadrant is most susceptible to damage and may be the earliest and dominant manifestation of visual dysfunction [33]. While the exact cause of this phenomenon is unclear, one plausible explanation is that the temporal RNFL quadrant is made up primarily of small-sized parvocellular axons inside the papillomacular bundle. Whether the smaller axons are more prone to damage is unknown, but they may have a less efficient remyelination process than larger axon [34]. Moreover, according to previous studies, temporal RNFL has a different developmental pattern from other quadrants. The expansion of the sclera during this development causes the nerve fibers to be distributed over a wider surface area, resulting in the early thinning of temporal RNFL. This pattern does not occur in the nasal quadrant where the thickness is higher [35].

We also found significantly delayed P100 latency in MS patients, as has been reported in previous studies [11, 36, 37]. Compared to MRI, VEP is more sensitive in detecting optic nerve demyelination but not sensitive enough to confirm MS diagnosis. In suspected cases of MS without evidence of ON, delayed latency of P100 in VEP may indicate optic nerve demyelination and suggest the incident of MS [38].

Compared with healthy individuals, MS patients have a significantly higher rate of optic atrophy. In certain cases, optic atrophy may result from a previous severe inflammation that occurs diffusely or temporally [39].

4.2. Evaluation of Visual Parameters in MS Group over 1 Year. As discussed by Chatziralli et al., visual acuity is restored almost completely within 6 months after an ON episode [11]. Similarly, contrast sensitivity showed equal improvement in 6-9 months after the conduction block caused by edema and 
TABLE 5: Comparison of GCIPL and RNFL thickness between RRMS and SPMS eyes.

\begin{tabular}{|c|c|c|c|c|c|}
\hline Variable & $n$ & RRMS & $n$ & SPMS & $p$ \\
\hline \multicolumn{6}{|c|}{ Mean \pm standard deviation } \\
\hline Mean & 42 & $73.19 \pm 10.32$ & 12 & $62.83 \pm 7.31$ & $0.002^{\mathrm{a}}$ \\
\hline Superior & 42 & $73.64 \pm 10.96$ & 12 & $62.25 \pm 8.50$ & $0.002^{b}$ \\
\hline Superonasal & 42 & $73.81 \pm 11.98$ & 12 & $61.17 \pm 9.16$ & $0.002^{\mathrm{a}}$ \\
\hline Inferonasal & 42 & $73.14 \pm 12.56$ & 12 & $60.25 \pm 7.26$ & $0.003^{\mathrm{a}}$ \\
\hline Inferior & 42 & $71.98 \pm 10.95$ & 12 & $60.17 \pm 4.93$ & $0.001^{\mathrm{a}}$ \\
\hline Inferotemporal & 42 & $73.98 \pm 9.96$ & 12 & $64.33 \pm 6.72$ & $0.003^{\mathrm{a}}$ \\
\hline Superotemporal & 42 & $73.29 \pm 9.22$ & 12 & $63.67 \pm 8.32$ & $0.002^{b}$ \\
\hline \multicolumn{6}{|l|}{ RNFL } \\
\hline Mean & 42 & $91.79 \pm 13.88$ & 12 & $76.92 \pm 7.00$ & $<0.001^{\mathrm{b}}$ \\
\hline Superior & 42 & $119.43 \pm 22.02$ & 12 & $102.58 \pm 16.50$ & $0.018^{b}$ \\
\hline Nasal & 42 & $69.33 \pm 11.05$ & 12 & $64.08 \pm 7.93$ & $0.132^{\mathrm{b}}$ \\
\hline Inferior & 42 & $115.88 \pm 19.64$ & 12 & $90.67 \pm 10.22$ & $<0.001^{\mathrm{a}}$ \\
\hline Temporal & 42 & $61.93 \pm 17.42$ & 12 & $50.42 \pm 10.93$ & $0.035^{\mathrm{b}}$ \\
\hline
\end{tabular}

GCIPL: ganglion cell inner plexiform layer; RNFL: retinal nerve fiber layer, RRMS: relapsing-remitting multiple sclerosis; SPMS: secondary progressive multiple sclerosis. ${ }^{\mathrm{a}}$ Mann-Whitney test, ${ }^{\mathrm{b}} \mathrm{T}$-independent test.

TABle 6: Profile of MS eyes that developed optic atrophy at 6 months based on the history of ON.

\begin{tabular}{|c|c|c|c|c|c|c|}
\hline Eyes code & 024 & 069 & 071 & 072 & 113 & 114 \\
\hline History of ON & Yes & Yes & No & No & No & No \\
\hline Sex & Female & Female & Female & Female & Female & Female \\
\hline Age (years) & 29 & 32 & 47 & 47 & 27 & 27 \\
\hline MS subtype & RRMS & RRMS & RRMS & RRMS & RRMS & RRMS \\
\hline Disease duration (years) & 6.29 & 0.75 & 0.5 & 0.5 & 3 & 3 \\
\hline Number of relapses & 6 & 3 & 0 & 0 & 6 & 6 \\
\hline EDSS & 1.5 & 3.0 & 1.0 & 1.0 & 6.0 & 6.0 \\
\hline BCVA (logmar) & 0.00 & 0.00 & 0.00 & 0.00 & 0.00 & 0.00 \\
\hline Contrast sensitivity & 1.65 & 1.95 & 1.65 & 1.65 & 1.95 & 1.95 \\
\hline P100 latency & 115.30 & 103.15 & 104.30 & 110.85 & 163.60 & 125.60 \\
\hline \multicolumn{7}{|l|}{ GCIPL } \\
\hline Mean & 62.00 & 84.00 & 78.00 & 80.00 & 72.00 & 75.00 \\
\hline Superior & 65.00 & 87.00 & 77.00 & 79.00 & 73.00 & 73.00 \\
\hline Superonasal & 66.00 & 87.00 & 80.00 & 80.00 & 73.00 & 72.00 \\
\hline Inferonasal & 64.00 & 82.00 & 80.00 & 84.00 & 75.00 & 73.00 \\
\hline Inferior & 56.00 & 82.00 & 77.00 & 79.00 & 69.00 & 78.00 \\
\hline Inferotemporal & 60.00 & 85.00 & 76.00 & 81.00 & 71.00 & 78.00 \\
\hline Superotemporal & 64.00 & 82.00 & 75.00 & 75.00 & 69.00 & 74.00 \\
\hline \multicolumn{7}{|l|}{ RNFL } \\
\hline Mean & 79.00 & 106.00 & 93.00 & 96.00 & 93.00 & 90.00 \\
\hline Superior & 115.00 & 132.00 & 126.00 & 132.00 & 106.00 & 110.00 \\
\hline Nasal & 62.00 & 71.00 & 61.00 & 68.00 & 68.00 & 73.00 \\
\hline Inferior & 93.00 & 143.00 & 123.00 & 128.00 & 109.00 & 108.00 \\
\hline Temporal & 46.00 & 78.00 & 61.00 & 55.00 & 87.00 & 71.00 \\
\hline
\end{tabular}

ON: optic neuritis; EDSS: Expanded Disability Status Scale.

inflammation has cleared up [40]. In the present study, we excluded patients with a history of ON within 6 months prior to enrollment which explains why we did not obtain a significant difference in visual acuity and contrast sensitivity in the MS group throughout the study.

The VEP results of our MS participants are in line with previous studies that reported lengthening of P100 latency 3 months after an acute episode and lasted for 1 year [40, 41]. Another study suggests that VEP abnormality may still be detected after $\geq 1$ year but the rates of deceleration will decrease [42].

In our analysis, the average value of GCIPL along with the inferior, inferotemporal, inferonasal, and superonasal quadrant in MS patients decreased significantly over 1 year. This finding supported other studies by Pillay [31] and Rebodella [43], who also reported that the supero- and inferonasal quadrants had the highest abnormality rate. As discussed by Shi et al. [44], the thinning zone occurs predominantly at 
nasal $1.98 \mathrm{~mm}$ and inferior $0.42 \mathrm{~mm}$ from the fovea with a circular area (diameter $=1 \mathrm{~mm}$ ), called the $\mathrm{M}$ zone (for MS). The $\mathrm{M}$ zone may demonstrate the involvement of papillomacular bundle in the parvocellular pathway. It is true, according to pathological studies, that smaller axons are damaged in MS. Moreover, mitochondrial dysfunction also played a role in MS and the location of the M zone is similar to that of mitochondrial optic neuropathy [44].

In this study, we only found a significant RNFL thinning in the nasal quadrant between 0 - and 12-month measurement which is contradictory to previous studies [31, 45]. In a study by Garcia-Martin et al. [45], maximal RNFL loss was found in the superonasal and inferotemporal quadrant. In contrast, an improvement was observed in the superior and nasal quadrant between 0 and 6 months and 6 and 12 months, respectively. This increase is possibly due to astrogliosis which results in a false thickening of RNFL [18]. Thus, astrogliosis can impede the accuracy of OCT in measuring RNFL thickness.

We also observed an increase in the proportion of optic atrophy at 6 and 12 months. Regardless of the high dropout rate, at the 6 months alone, there were 6 eyes that developed optic atrophy. Hence, it is safe to say that the increased proportion was due to the progression of the disease itself, not attrition bias. The optic disc is the entry point of the optic nerve which is composed of numerous axons. In MS, axonal degeneration is responsible for the development of optic atrophy observed during funduscopy [39].

\subsection{Correlation between Visual Parameters, Disease Duration,} Number of Relapses, and Degree of Disability in MS Patients. Our analysis showed that GCIPL (superonasal, inferonasal quadrant) and mean RNFL had a negative correlation with the number of relapses. Likewise, GCIPL (mean, superonasal, inferonasal, inferior quadrant) and RNFL (inferior quadrant) demonstrated a negative correlation with the EDSS score as reported by previous studies [26, 32]. Pillay et al. [31] reported that RNFL $<88 \mu \mathrm{m}$ is associated with a threefold increase in the EDSS score in 3 years. Unlike previous studies, we found no correlation between OCT measures and disease duration $[11,21]$. However, P100 latency was significantly correlated with disease duration and the number of relapses. Bennetto et al. [22] also reported a similar finding but his study needs further statistical analysis to make a conclusion. According to the London Ontario cohort study, higher relapse frequency in the first 2 years is associated with a lower EDSS score [46].

Over 1-year evaluation, we found that GCIPL and RNFL alteration occurred at a more constant rate than P100 latency, both in the first 6 months or in the following months. Our findings appear contradictory with a study by Henderson et al. [21] which concluded that RNFL thinning does not follow a linear progression. They suggest that RNFL loss occurs faster in the early course of the disease when subclinical inflammatory demyelination takes place.
4.4. Differences of Visual Measures between MS Subtypes. We observed a significant difference in GCIPL and RNFL thickness between RRMS and SPMS at baseline. This finding indicates that neurodegeneration, marked by axonal and ganglion cell loss, occurs more rapidly in MS with progressive phase, regardless of disease duration, age, and previous ON history [47]. However, a different finding was reported by Balk et al. [20], in which a significant difference of RNFL (but not GCIPL) was observed between RRMS and progressive MS over 2 years. In other words, even though neuronal and axonal injury occurs during a relapse episode, only a few continue to deteriorate in the progressive phase [21]. In RRMS, the remitting phase provides the opportunity for the remyelination process to take place. However, in SPMS, demyelination or axonal degeneration happens without the remitting phase, supporting the fact that the damage caused in SPMS is more severe. Therefore, it can be seen at the beginning that the structure and function of the optic nerve in SPMS are clinically poorer than RRMS.

\subsection{History of Previous ON Influencing the Visual Parameters.} We also performed an adjusted analysis based on the patient's history of ON. In the MS-NO subgroup, GCIPL appeared to thicken over 12 months but a significant difference was found only in the inferonasal quadrant. Other parameters only showed subtle changes which may be due to the participants being in the remission phase and having no acute episode during the study [31]. Furthermore, if we look at the whole sample regardless of previous $\mathrm{ON}$, significant thinning was actually seen in every quadrant. Therefore, it can be concluded that the relatively stable visual measures among the MS-NO subgroup were because of insufficient sample size, assuming that participants who stayed in the study had thicker GCIPL than those who dropped out.

In contrast to MS-NO, the significant differences of OCT measures in the MS-NNO subgroup were possibly due to axonal loss resulting from previous subclinical ON that left a profound manifestation on the GCIPL [31]. Delayed P100 latency in this group corresponds with Pillay et al. who reported similar findings in eyes without a history of $\mathrm{ON}$ at 6 months [11]. In suspected MS cases, abnormality in VEP measurement illustrates the demyelinating process of the optic nerve and provides information about MS development [38].

\section{Conclusion}

In summary, this study showed that MS patients have lower visual acuity and contrast sensitivity, thinner GCIPL and RNFL thickness (except in the nasal quadrant), and longer P100 latency compared with healthy individuals. GCIPL and RNFL thinning occurs at 6 and 12 months but does not correlate with disease duration, the number of relapses, and EDSS score. The thinning of GCIPL from 0 to 12 months is more prominent compared with RNFL, and it is shown in 
the inferonasal, inferior, and inferior temporal quadrant. The thinning of GCIPL and RNFL is worse in SPMS than in RRMS.

5.1. Strength and Limitation. In Indonesia, this is the first longitudinal study of multiple sclerosis in which the data on MS are still scarce. We recruited healthy controls with matching age and sex with the MS group. The optic nerve was evaluated in various parameters to give more comprehensive results. However, our study has potential limitations, including small sample size (which partly due to the rarity of MS itself) and a relatively short follow-up period. Since it is a cohort study, dropout was inevitable. Data regarding disease duration and relapse frequency were obtained through selfreporting which possibly contributed to recall bias. We also did not analyze factors affecting disability, such as the treatments received during the study. Lastly, our study needs further statistical analysis with Generalized Estimating Equations (GEEs) to identify the correlation between disease duration, the number of relapses, EDSS, and the structure and function of the optic nerve.

\section{Data Availability}

The data that support the findings of this study are available from the corresponding author upon reasonable request.

\section{Ethical Approval}

This study was approved by the Ethics Committee, Faculty of Medicine Universitas Indonesia. All human research procedures were followed in accordance with the ethical standards of the committee responsible for human experimentation (institutional and national) and with the Helsinki Declaration of 1975, as revised in 2013.

\section{Consent}

Participants had given written consent before taking part in the study and had the right to refuse to participate without penalty if they wish.

\section{Conflicts of Interest}

The authors declare that there are no conflicts of interest regarding the publication of this paper.

\section{Acknowledgments}

The authors would like to thank Yayasan Multipel Sklerosis Indonesia (YMSI) for participating in this study. This study was funded by Hibah PITTA 2018 by DRPM Universitas Indonesia (PITTA/214/FK/2018).

\section{References}

[1] S. Eskandarieh, P. Heydarpour, A. Minagar, S. Pourmand, and M. A. Sahraian, "Multiple sclerosis epidemiology in east asia, south east asia and south asia: a systematic review," Neuroepidemiology, vol. 46, no. 3, pp. 209-221, 2016.
[2] W. Kusumadewi, D. Imran, F. Witjaksono et al., "Low vitamin $\mathrm{D}-25(\mathrm{OH})$ level in Indonesian multiple sclerosis and neuromyelitis optic patients," Multiple Sclerosis and Related Disorders, vol. 25, pp. 329-333, 2018.

[3] A. Ascherio and K. Munger, "Epidemiology of multiple sclerosis: from risk factors to prevention," Seminars in Neurology, vol. 28, no. 1, pp. 17-28, 2008.

[4] R. Milo and E. Kahana, "Multiple sclerosis: geoepidemiology, genetics and the environment," Autoimmunity Reviews, vol. 9, no. 5, pp. A387-A394, 2010.

[5] M. Kolappan, A. P. D. Henderson, T. M. Jenkins et al., "Assessing structure and function of the afferent visual pathway in multiple sclerosis and associated optic neuritis," Journal of Neurology, vol. 256, no. 3, pp. 305-319, 2009.

[6] F. Costello, "The afferent visual pathway: designing a structural-functional paradigm of multiple sclerosis," ISRN Neurology, vol. 2013, pp. 1-17, 2013.

[7] S. Saidha, S. B. Syc, M. A. Ibrahim et al., "Primary retinal pathology in multiple sclerosis as detected by optical coherence tomography," Brain, vol. 134, no. 2, pp. 518-533, 2011.

[8] M. Adhi and J. S. Duker, "Optical coherence tomographycurrent and future applications," Current Opinion in Ophthalmology, vol. 24, no. 3, pp. 213-221, 2013.

[9] J. Bennett, J. De Seze, M. Lana-Peixoto et al., "Neuromyelitis optica and multiple sclerosis: seeing differences through optical coherence tomography," Multiple Sclerosis Journal, vol. 21, no. 6, pp. 678-688, 2015.

[10] S. Noval, I. Contreras, S. Muñoz, C. Oreja-Guevara, B. Manzano, and G. Rebolleda, "Optical coherence tomography in multiple sclerosis and neuromyelitis optica: an update," Multiple Sclerosis International, vol. 2011, Article ID 472790, 11 pages, 2011.

[11] I. P. Chatziralli, M. M. Moschos, D. Brouzas, K. Kopsidas, and I. D. Ladas, "Evaluation of retinal nerve fibre layer thickness and visual evoked potentials in optic neuritis associated with multiple sclerosis," Clinical and Experimental Optometry, vol. 95, no. 2, pp. 223-228, 2012.

[12] L. J. Balcer and E. M. Frohman, "Evaluating loss of visual function in multiple sclerosis as measured by low-contrast letter acuity," Neurology, vol. 74, no. 17, pp. S16-S23, 2010.

[13] S. B. Syc, S. Saidha, S. D. Newsome et al., "Optical coherence tomography segmentation reveals ganglion cell layer pathology after optic neuritis," Brain, vol. 135, no. 2, pp. 521-533, 2012.

[14] C. Oreja-Guevara, S. Noval, J. Alvarez-Linera et al., "Clinically isolated syndromes suggestive of multiple sclerosis: an optical coherence tomography study," PLoS One, vol. 7, no. 3, Article ID e33907, 2012.

[15] R. S. Maldonado, P. Mettu, M. El-Dairi, and M. T. Bhatti, “The application of optical coherence tomography in neurologic diseases," Neurology: Clinical Practice, vol. 5, no. 5, pp. 460-469, 2015.

[16] F. Costello, S. Coupland, W. Hodge et al., "Quantifying axonal loss after optic neuritis with optical coherence tomography," Annals of Neurology, vol. 59, no. 6, pp. 963-969, 2006.

[17] Y.-M. Huang-Link, M. Fredrikson, and H. Link, "Benign multiple sclerosis is associated with reduced thinning of the retinal nerve fiber and ganglion cell layers in non-opticneuritis eyes," Journal of Clinical Neurology, vol. 11, no. 3, p. 241, 2015.

[18] S. Saidha, S. B. Syc, M. K. Durbin et al., "Visual dysfunction in multiple sclerosis correlates better with optical coherence tomography derived estimates of macular ganglion cell layer 
thickness than peripapillary retinal nerve fiber layer thickness," Multiple Sclerosis Journal, vol. 17, no. 12, pp. 1449-1463, 2011.

[19] D. M. Movassat, D. N. Piri, and D. M. N. AhmadAbadi, "Visual evoked potential study in multiple sclerosis disease," Iranian Journal of Ophthalmology, vol. 21, no. 4, pp. 37-44, 2009.

[20] L. J. Balk, A. Cruz-Herranz, P. Albrecht et al., "Timing of retinal neuronal and axonal loss in MS: a longitudinal OCT study," Journal of Neurology, vol. 263, no. 7, pp. 1323-1331, 2016.

[21] A. P. D. Henderson, S. A. Trip, P. G. Schlottmann et al., “An investigation of the retinal nerve fibre layer in progressive multiple sclerosis using optical coherence tomography," vol. 131, no. Pt 1, pp. 277-287, 2007.

[22] L. Bennetto, J. Burrow, H. Sakai, J. Cobby, N. Robertson, and N. Scolding, "The relationship between relapse, impairment and disability in multiple sclerosis," Multiple Sclerosis Journal, vol. 17, no. 10, pp. 1218-1224, 2011.

[23] T. Oberwahrenbrock, S. Schippling, M. Ringelstein et al., "Retinal damage in multiple sclerosis disease subtypes measured by high-resolution optical coherence tomography," Multiple Sclerosis International, vol. 2012, Article ID 530305, 10 pages, 2012.

[24] A. Soler García, A. González Gómez, L. C. Figueroa-Ortiz, A. García-Ben, and J. García-Campos, "Relationship between contrast sensitivity test and disease severity in multiple sclerosis patients," Archivos de la Sociedad Española de Oftalmología (English Edition), vol. 89, no. 9, pp. 347-351, 2014.

[25] R. S. Maldonado, P. Mettu, M. El-Dairi, and M. T. Bhatti, “The application of optical coherence tomography in neurologic diseases," Neurology: Clinical Practice, vol. 5, no. 5, pp. 460-469, 2015.

[26] J. Britze and J. L. Frederiksen, "Optical coherence tomography in multiple sclerosis,” Eye, vol. 32, no. 5, pp. 884-888, 2018.

[27] G. Bsteh, H. Hegen, B. Teuchner et al., "Peripapillary retinal nerve fibre layer as measured by optical coherence tomography is a prognostic biomarker not only for physical but also for cognitive disability progression in multiple sclerosis," Multiple sclerosis (Houndmills, Basingstoke, England), vol. 25, no. 2, pp. 196-203, 2017.

[28] R. A. Arpandy, "Differences in structure and function of optical nerve in multiple sclerosis and neuromyelitis optica spectrum disorders and its relation to degree of disability, duration of disease, and number of relapse," 2017, http:// research.fk.ui.ac.id/borangmhs/index.php/list-borangmahasiswa $1 /$ details/2/88.

[29] A. Wijaya, "Normative values of visual evoked potentials' latencies and amplitudes in adults," Neurona, vol. 35, no. 2, pp. 78-85, 2018.

[30] M. L. McHugh, "Interrater reliability: the kappa statistic," Biochemia Medica, pp. 276-282, 2012.

[31] G. Pillay, A. Ganger, D. Singh et al., "Retinal nerve fiber layer and ganglion cell layer changes on optical coherence tomography in early multiple sclerosis and optic neuritis cases," Indian Journal of Ophthalmology, vol. 66, no. 1, pp. 114-119, 2018.

[32] J. N. Ratchford, S. Saidha, E. S. Sotirchos et al., "Active MS is associated with accelerated retinal ganglion cell/inner plexiform layer thinning," Neurology, vol. 80, no. 1, pp. 47-54, 2012.

[33] D. L. Budenz, D. R. Anderson, R. Varma et al., "Determinants of normal retinal nerve fiber layer thickness measured by stratus OCT," Ophthalmology, vol. 114, no. 6, pp. 1046-1052, 2007.

[34] J. M. Gelfand, D. S. Goodin, W. J. Boscardin, R. Nolan, A. Cuneo, and A. J. Green, "Retinal axonal loss begins early in the course of multiple sclerosis and is similar between progressive phenotypes," PLoS One, vol. 7, no. 5, Article ID e36847, 2012.

[35] A. Patel, R. Purohit, H. Lee et al., "Optic nerve head development in healthy infants and children using handheld spectral-domain optical coherence tomography," Ophthalmology, vol. 123, no. 10, pp. 2147-2157, 2016.

[36] A. A. Thamer, N. H. Mohammed, and A. M. Ibrahim, "Role of visual evoked potentials in multiple sclerosis," Journal of the Faculty of Medicine, Baghdad, vol. 56, 2014.

[37] R. Pul, M. Saadat, F. Morbiducci et al., "Longitudinal timedomain optic coherence study of retinal nerve fiber layer in IFN $\beta$-treated and untreated multiple sclerosis patients," Experimental and Therapeutic Medicine, vol. 12, no. 1, pp. 190-200, 2016.

[38] N. Chirapapaisan, S. Laotaweerungsawat, W. Chuenkongkaew et al., "Diagnostic value of visual evoked potentials for clinical diagnosis of multiple sclerosis," Documenta Ophthalmologica, vol. 130, no. 1, pp. 25-30, 2014.

[39] P. Francisco and G.-V. Lorena, "Diagnosis approach of optic neuritis," Journal of Neurology \& Neurophysiology, vol. 6, no. 6, 2015.

[40] A. Brusa, S. J. Jones, and G. T. Plant, "Long-term remyelination after optic neuritis," Brain, vol. 124, no. 3, pp. 468-479, 2001.

[41] R. Herrero, E. Garcia-Martin, C. Almarcegui et al., "Progressive degeneration of the retinal nerve fiber layer in patients with multiple sclerosis," Investigative Opthalmology \& Visual Science, vol. 53, no. 13, p. 8344, 2012.

[42] M. A. Hely, P. G. McManis, J. C. Walsh, and J. G. McLeod, "Visual evoked responses and ophthalmological examination in optic neuritis," Journal of the Neurological Sciences, vol. 75, no. 3, pp. 275-283, 1986.

[43] G. Rebolleda, L. Diez-Alvarez, A. Casado et al., "OCT: new perspectives in neuro-ophthalmology," Saudi Journal of Ophthalmology, vol. 29, no. 1, pp. 9-25, 2015.

[44] C. Shi, H. Jiang, G. R. Gameiro et al., "Visual function and disability are associated with focal thickness reduction of the ganglion cell-inner plexiform layer in patients with multiple sclerosis," Investigative Opthalmology \& Visual Science, vol. 60, no. 4, p. 1213, 2019.

[45] E. Garcia-Martin, J. R. Ara, J. Martin et al., "Retinal and optic nerve degeneration in patients with multiple sclerosis followed up for 5 years," Ophthalmology, vol. 124, no. 5, pp. 688-696, 2017.

[46] A. Scalfari, A. Neuhaus, A. Degenhardt et al., "The natural history of multiple sclerosis, a geographically based study 10 : relapses and long-term disability," Brain, vol. 133, no. 7, pp. 1914-1929, 2010.

[47] R. Behbehani, A. Abu Al-Hassan, A. Al-Salahat, D. Sriraman, J. D. Oakley, and R. Alroughani, "Optical coherence tomography segmentation analysis in relapsing remitting versus progressive multiple sclerosis," PLoS One, vol. 12, no. 2, Article ID e0172120, 2017.

[48] F. Costello, W. Hodge, Y. I. Pan, M. Freedman, and C. DeMeulemeester, "Differences in retinal nerve fiber layer atrophy between multiple sclerosis subtypes," Journal of the Neurological Sciences, vol. 281, no. 1-2, pp. 74-79, 2009. 